\title{
Dual-Polarized Wearable Antenna/Rectenna for Full-Duplex and MIMO Simultaneous Wireless Information and Power Transfer (SWIPT)
}

\author{
Mahmoud Wagih, Member, IEEE, Geoffrey S. Hilton, Alex S. Weddell, Member, IEEE, and \\ Steve Beeby, Senior Member, IEEE
}

\begin{abstract}
Owing to the mobility of a wearable antenna and the unpredictable body-centric communications environment, dualpolarization antennas are essential for both communications and energy harvesting. This paper presents a dual-polarized four-port textile antenna/rectenna for wearable simultaneous wireless information and power transfer (SWIPT) applications. The proposed antenna utilizes dual ports for both off-body communication and energy harvesting from horizontal and vertical polarizations. The antenna maintains a $100 \mathrm{MHz}$ bandwidth with an $S_{11}$ under $-10 \mathrm{~dB}$ around $2.4 \mathrm{GHz}$ in the presence and absence of the human body, and at least $10 \mathrm{~dB}$ small-signal and large-signal isolation between all ports. The antenna maintains a 70-88\% measured total efficiency and 8.4-9.6 dBi gain for various on-phantom positions across both communication ports. The measured mutual coupling is under $-10 \mathrm{~dB}$ between copolarized rectenna/antenna ports, and under $-16 \mathrm{~dB}$ between orthogonally-polarized ports. A high RF to DC peak power conversion efficiency of over $70 \%( \pm 5 \%)$ is achieved with a broadside harvesting pattern. Based on the proposed antenna's performance, SWIPT microstrip antennas can be adopted for both full-duplex and MIMO applications, significantly reducing the complexity of future battery-free networks for both wearable and non-wearable applications.
\end{abstract}

Index Terms-Antennas, Body Area Networks, E-Textiles, Microstrip Antennas, Microwave power transmission, Multi-In Multi-Out (MIMO) Antennas, Rectennas, Rectifiers, Wearable Antennas.

\section{INTRODUCTION}

$\mathbf{S}$ IMULTANEOUS wireless information and power transfer (SWIPT) has attracted significant research interest for sustainable Internet of Things (IoT) networks [1]. In a SWIPT network, a basestation transmits single or multiple waveforms carrying both information and power to wireless "edge" nodes. The nodes would typically utilize multiple antennas to receive the power and decode information. Alternatively, for a single antenna, a complex transceiver architecture may be adopted to enable time-based scheduling of the harvesting/decoding, in

This work was supported by the UK Engineering and Physical Sciences Research Council (EPSRC) under Grant EP/P010164/1 and the European Commission through the project EnABLES, funded under H2020-EU.1.4.1.2 grant number: 730957. S. Beeby was supported by the Royal Academy of Engineering under the Chairs in Emerging Technologies scheme (Corresponding author: Mahmoud Wagih)

M. Wagih, A. S. Weddell, and S. Beeby are with the School of Electronics and Computer science, University of Southampton, Southampton, SO17 1BJ, U.K. (email:mahm1g15@ecs.soton.ac.uk)

G. S. Hilton is with the Communication Systems \& Networks Group, University of Bristol, Bristol BS8 1UB, U.K.

Digital Object Identifier: addition to hardware-based power-splitting. SWIPT has been extensively investigated for Multi-In Multi-Out (MIMO), fullduplex, and body area networks applications [1]. Nevertheless, the literature on SWIPT antennas is limited and antenna-based solutions for SWIPT have not been widely investigated. As a result, power splitting is often assumed in studies involving full-duplex or MIMO data transmission [2].

Wireless power transmission (WPT) and subsequently Radio Frequency Energy Harvesting (RFEH) have been explored for wearable applications, with an array of textile-based antenna and rectenna implementations from UHF to mmWave bands [3]-[9]. Textile rectennas for WPT and RFEH leverage the extensive research on wearable antennas for off-body communications, as power is often received from a basestation placed off the body [10]. Off-body antennas have been developed as part of multi-mode on/in-body antennas [11], [12]. In addition, dual-port textile-based microstrip patch antennas have been proposed with high-isolation for full-duplex applications [13]. Dual-polarization multi-port antennas with high port isolation are beneficial for various applications such as full-duplex and MIMO communications [13]-[17].

Until recently, no antenna designs have been dedicated to SWIPT [18]. In [19], a dual-port $50 \Omega$ antenna printed on a 3D printed enclosure was connected to both a rectifier and a transceiver. A hybrid RFID/Schottky dual-polarized rectenna was also proposed for simultaneous harvesting and sensing [20]. A dual-port antenna was proposed in [21] with a PIN diode for frequency re-configurable operation as a rectenna and an antenna. Hybrid couplers were later investigated for SWIPT in [22], but their large footprint adds to the system's complexity and restricts the implementation to low-loss RF substrates to avoid the insertion losses. Furthermore, [19], [21], [22] are not different from standard dual-port $50 \Omega$ antennas, and rely on an external rectifier matching network. Recently, a triple-band coupled resonator was proposed for SWIPT applications, RF-DC performance as well as power splitting mechanisms have not been investigated [23].

In [24], we first proposed a dual-port $2.4 \mathrm{GHz}$ textile antenna based on antenna-rectifier co-design for wearable applications. In [18], antenna rectifier co-design was utilized to implement a sub-1 $\mathrm{GHz}$ rectenna within a $2.4 \mathrm{GHz}$ off-body textile patch for dual-band/mode SWIPT. Nevertheless, both implementations, [24] and [18], maximize the isolation by designing the antenna and rectenna ports with orthogonal polarizations. In the context of wearable WPT and communications, 


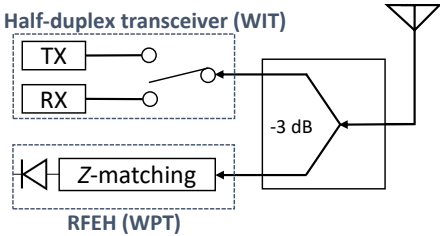

(a) Conventional half-duplex comms+power-splitting SWIPT

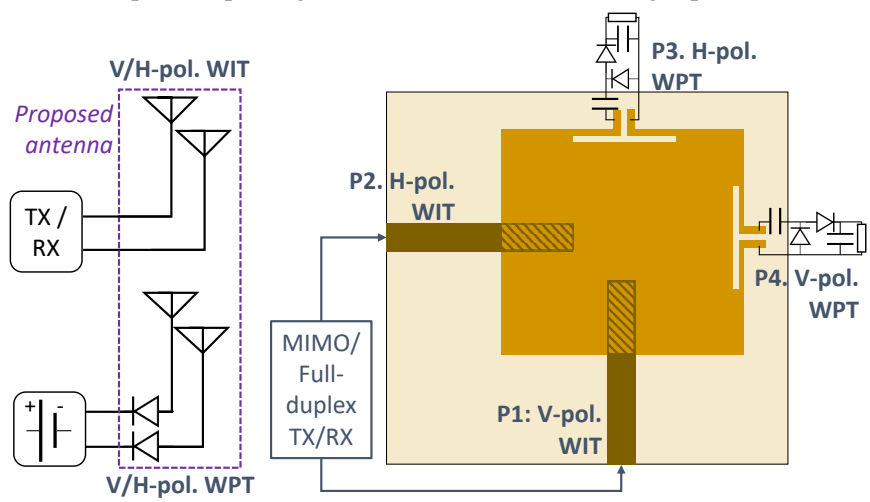

(c) Proposed antenna for MIMO/full-duplex SWIPT

Fig. 1. Examples of multi-antenna SWIPT systems: (a) conventional halfduplex TX/RX with power splitting for single-pol. WPT; (b) dual-antenna MIMO with a separate WPT antenna; (c) the proposed antenna.

polarization alignment between an off-body basestation and the wearable antenna is unlikely due to the mobility of the user, where polarization mismatch can cause up to $37.5 \mathrm{~dB}$ channel loss in line-of-sight links [25]. While a dual-polarization WPTonly rectenna was proposed to mitigate this issue [3], the effect of simultaneous WPT on dual-polarized communications, e.g. MIMO or full-duplex, is unknown.

In this paper, a four-port dual-polarized antenna/rectenna is proposed for SWIPT applications, utilizing antenna-rectifier co-design within a textile microstrip patch. The key contributions in this work can be summarized as:

1) Demonstrating the first dual-polarized four-port antenna/rectenna, for full-duplex/MIMO SWIPT;

2) Identifying a non-linear power dependency in the communications port isolation despite the antenna's apparent passiveness due to the rectifiers' presence;

3) Achieving higher port isolation than conventional microstrip and probe feeds between both the communications and power harvesting ports in small and largesignal operation.

\section{Dual-Polarization SWIPT Antenna Design}

\section{A. Multi-Port Antennas for MIMO and Full-Duplex SWIPT}

Examples of multi-antenna systems include MIMO and fullduplex communications, where high isolation between the antenna elements is essential. In addition, SWIPT can be regarded as a multi-antenna system should the wireless information transfer (WIT) and the wireless power transmission (WPT) utilize separate antennas, or as in [18] a dual-port antenna with high port isolation. Fig. 1-a shows an example of a single antenna for SWIPT, where a power divider is used to split a the incident radiation between the receiver $(\mathrm{RX})$ and the rectifier. Inside the transceiver, an RF switch is used for time-based division between transmission and reception using the shared antenna [13]. In addition, the rectifier must use a standalone impedance matching network to transform the power divider's $50 \Omega$ impedance to the rectifier's [26]. Fig. 1b shows an alternative example where multiple antennas may be used. Two antennas, typically with orthogonal polarizations [14], are used for a MIMO or a full-duplex transceiver. A separate antenna is used for WPT where a low mutual coupling is essential between the WPT and TX/RX antennas. Both systems in Fig. 1-a and b can only harvest power from a single polarization and will need a separate antenna or a dualpolarized rectenna, [3], [7], for polarization-independent WPT.

Four-port antennas with a shared radiation aperture have been proposed for MIMO WIT [27] and for WPT applications [28]. A four-port offset-fed patch antenna was proposed for matching network-free WPT [28]. However, due to the tilted geometry of the patch and the offset feed position, such a design may not be suitable for communications due to the difficulty of achieving a 50\% match. As for the MIMO structure in [27], the ports are matched to $50 \%$ and, owing to their single-ended topology, will require an impedance matching network to connect to a rectifier. Therefore, the proposed hybrid feeding mechanism is essential for efficient and scalable matching network-free SWIPT, which can be used to power MIMO and SWIPT systems

Fig. 1-c shows the equivalent schematic and layout of the proposed multi-port antenna. With two orthogonal polarization ports for both WPT and wireless information transfer (WIT), and high isolation between the ports, the antenna could be considered as a replacement for a four-antenna system. In addition, the input impedance of the WPT ports can be tuned to varying complex impedances, enabling the antenna to directly match the rectifier, thus eliminating the matching network stage in Fig. 1-a and b. However, the performance of such a multi-port antenna will be highly dependant on the isolation, which can be implemented through the feed design.

To illustrate the power reception mechanism of the proposed shared aperture patch, Fig. 2 shows the power received by each port from an incident power density $S$, for linear (a) and circular (b) polarizations. $A_{\text {eff }}$ is the effective area of the antenna, for each receiving port, given by

$$
A_{\mathrm{eff}}=\frac{\lambda^{2}}{4 \pi} G_{\mathrm{RX}} .
$$

where $G_{\mathrm{RX}}$ is the realized gain, inclusive of impedance mismatch and mutual coupling losses.

As observed in Fig. 2-a, a linearly polarized $S$ will be split between the dual-polarized ports, based on the realized gain of each port. As both ports are linearly polarized and achieve high polarization purity, demonstrated in Section III$\mathrm{C}$, both the WPT and WIT ports will be able to discriminate between the incident polarizations, allowing up-link and downlink users to be selectively-powered by the base-station [1]. As for a circularly-polarized wave, in Fig. 2-b, the power is expected to be received over all ports with equal division 
(a)

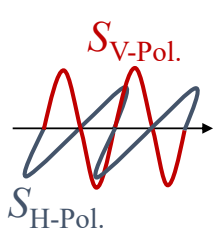

(b)

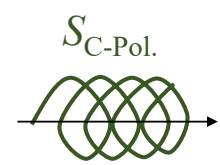

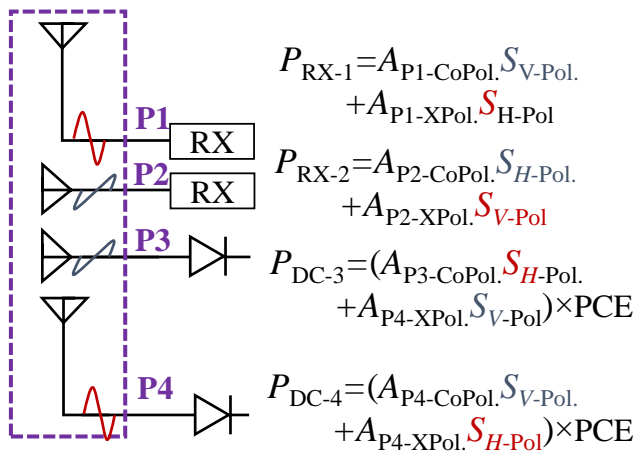

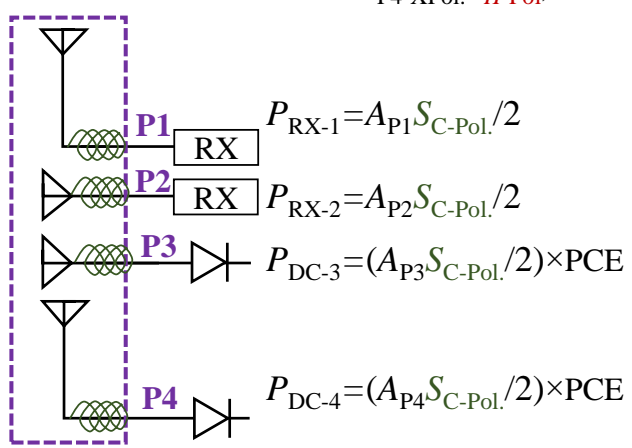

Fig. 2. Power reception mechanism of the proposed antenna for: (a) linearly polarized $S$, and (b) circularly polarized $S$.

between the ports, with a $-3 \mathrm{~dB}$ polarization mismatch loss due to receiving a circulary polarized wave using a linearlypolarized receiver [26], [29]. However, this eliminates the angular dependence for a given angular alignment, making circularly-polarized WPT excitations a more resilient option to channel misalignments.

At a system level, several components can be utilize WPT and SWIPT in MIMO and full-duplex systems. At a networklevel the proposed multi-port antenna could be regarded as a multi-antenna SWIPT system; SWIPT networks using multiple antennas, as opposed to switching and splitting, are typically found to reduce interference and improve the overall network throughput [1]. In addition. To help overcome the mutual coupling problem and "noise" generated form the colocated high-power ( -20 to $0 \mathrm{dBm}$ ) WPT, relative to the low power communications (sub $-60 \mathrm{dBm}$ ), several low-power noise-cancellation have been proposed. For example, the selfinterference cancellation front-end proposed in [30] for fullduplex communication does not add to the power consumption of the transceiver while offering over $50 \mathrm{~dB} T X / \mathrm{RX}$ isolation, making it highly suitable for WPT and RFEH systems.

\section{B. Feeding Mechanism for SWIPT}

Textile-based microstrip patch antennas have been demonstrated using a variety of feeding mechanisms. The simplest and most common is the probe-fed patch, shown in Fig. 3-a, most suited for coaxial interconnects and circuitry positioned behind the antenna. An inset microstrip feed (Fig. 3-b) or a proximity-coupled microstrip (Fig. 3-c) can be used for planar components such as a microstrip PCB rectifier [5]. The resulting input impedance is predominantly resistive at resonance and can be tuned to match $50 \Omega$ by changing the

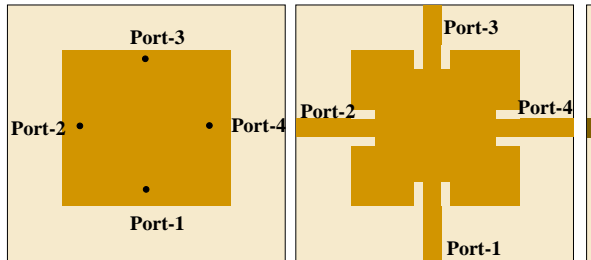

(a) Probe feed

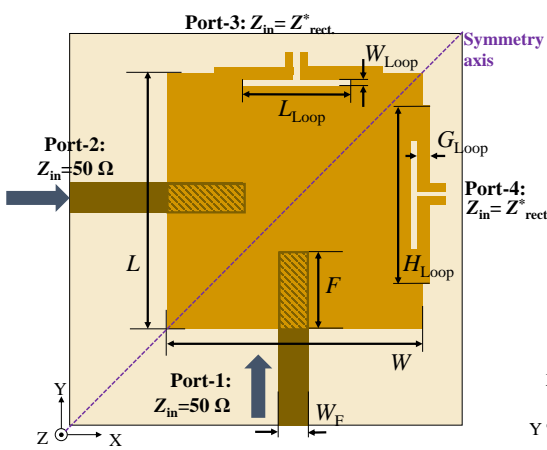

(d) Proposed dual-polarization proximity/inductive-fed microstrip patch

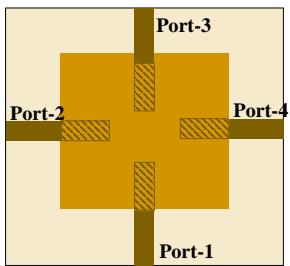

(c) Proximity-coupling feed

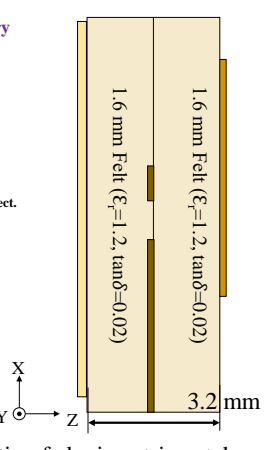

Fig. 3. Feeding mechanisms for a four-port SWIPT microstrip patch. (a) Probe-feed; (b) inset microstrip feed; (c) proximity-coupled microstrip feed; (d) proposed hybrid proximity coupling and complex- $Z$ loop feeds layout and cross-section.

feed point. For a multi-port SWIPT rectenna, a high isolation between the ports is the key figure-of-merit.

The aforementioned feeding structures have been simulated in CST Microwave Studio for a $53 \times 53 \mathrm{~mm}$ patch over a $3.2 \mathrm{~mm}$-thick felt substrate $\left(\epsilon_{r}=1.2, \tan \delta=0.02\right)$. Fig. 4 shows the simulated mutual coupling between the ports. The WIT/WIT coupling represents the mutual coupling between the orthogonal communications ports $\left(S_{21}\right)$, which has been reported as needing to be under $15 \mathrm{~dB}$ for MIMO or fullduplex applications [13]. As for the WPT/WIT coupling $\left(S_{41}\right)$, this represents the coupling between the co-polarized rectifier/transceiver ports, which can degrade the power harvesting capabilities of the rectenna.

From Fig. 4, the high coupling between the probe-fed and inset-fed ports indicates their unsuitability for a dualpolarization SWIPT patch. Proximity coupling on the other hand improves the WIT/WIT port isolation at $2.4 \mathrm{GHz}$ to $20 \mathrm{~dB}$, matching that of novel feeding mechanisms such as the strip-loaded microstrip lines [13]. Inductive feeds have been proposed for RFID ICs (i.e. on-chip CMOS charge pump) or Schottky-based rectenna applications [18], [20], to match the capacitive load impedance of rectifiers. The hybrid feeding mechanism, shown in Fig. 3-d, is proposed to present the rectifier ports with a complex impedance, eliminating the need for a matching network, and to improve the isolation between the WIT and WPT ports.

As observed in Fig. 4, the proposed feeding mechanism reduces the coupling between the co-polarized WIT/WPT ports $\left(S_{41}\right)$ by $1 \mathrm{~dB}$ compared to the proximity coupling feed, and over $2 \mathrm{~dB}$ compared to the probe feed. In addition, the proposed feed improves the isolation between the communications ports by $17 \mathrm{~dB}$, resulting in an $S_{21}<-35 \mathrm{~dB}$ at $2.4 \mathrm{GHz}$, when the WPT ports are normalized to the 


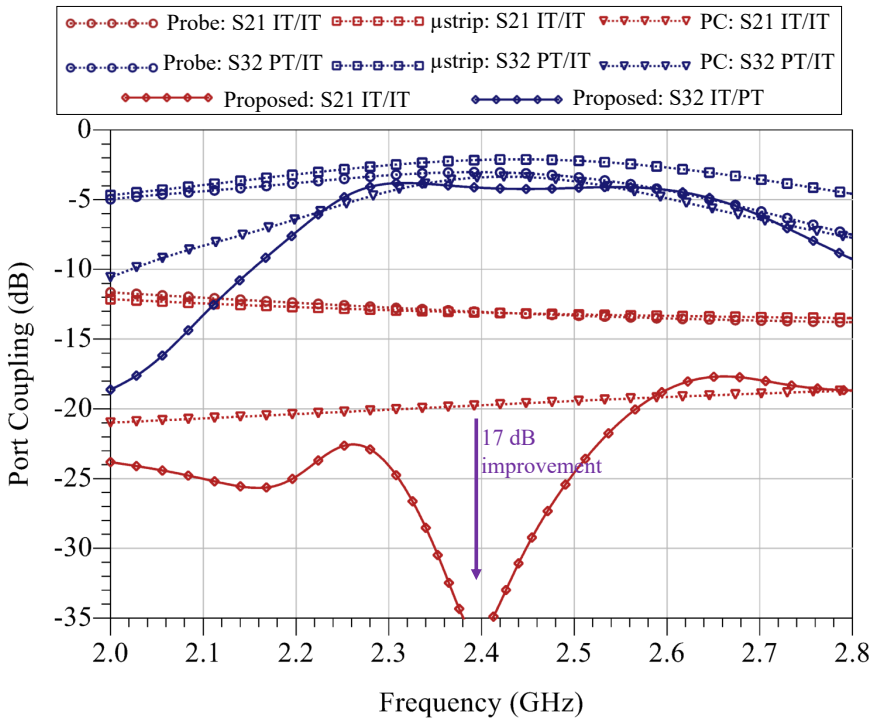

Fig. 4. Simulated mutual coupling between the V/H-pol. communications ports (WIT/WIT) and the co-polarized WPT/WIT ports (WPT/WIT), for the different feeds investigated; $\mu$ strip: inset feed; PC: proximity-coupled feed.

rectifiers' impedance. Therefore, it can be concluded that the presence of extra rectifier-loaded inductive-fed ports improves the isolation between orthogonally-polarized communication ports, showing its suitability for high isolation applications such as MIMO and full-duplex communications.

\section{Antenna/Rectenna Simulation and Tuning}

Based on the simulated high isolation, the hybrid inductive/proximity-coupled feeding mechanism is adopted as shown in Fig. 3-d. The resonance of the patch has been tuned by varying $L$ and $W . L=W=50 \mathrm{~mm}$ was chosen to maintain a minimum $S_{11}$ at $2.4 \mathrm{GHz}$. In order to tune $Z_{\text {in }}$ at the inductive port, the length of the loop $L_{\text {Loop }}$ is parametrically investigated. Fig. 5 shows the simulated $Z_{\text {in }}$ for varying $L_{\text {Loop }}$ over both ports.

As observed in Fig. 5-a, $Z_{\text {in }}$ is maintained around $50 \Omega$ for varying $L_{\mathrm{Loop}}$, showing that tuning the rectenna's $Z_{\text {in }}$ does not detune the WIT ports. In addition, Fig. 5-b demonstrates that the $Z_{\text {in }}$ magnitude can be tuned through $L_{\text {Loop }}$ without changing the resonant frequency. Fig. 6 shows that the antenna's WIT ports maintain their input bandwidth regardless of the loop's dimensions.

At $2.4 \mathrm{GHz}$, Fig. 7 shows that increasing $L_{\text {Loop }}$ linearly increases $\Im\left\{Z_{\text {in }}\right\}$, demonstrating good tunability for varying rectifier input impedances. This is particularly desirable should the antenna be matched to rectifiers based on different diode models, aimed at different power levels, or operating at different frequency bands [31]. Moreover, the simulated radiation efficiency of the rectenna is maintained above $63 \%$, in line with recent patch antenna implementations on the same felt substrate [5], [18].

Despite the differential complex- $Z$ feed of the antenna at the WPT ports, both the WPT and WIT ports radiate using the same mechanism. To explain, the shared-aperture between the four ports resonates at the first-order TM mode, TM01
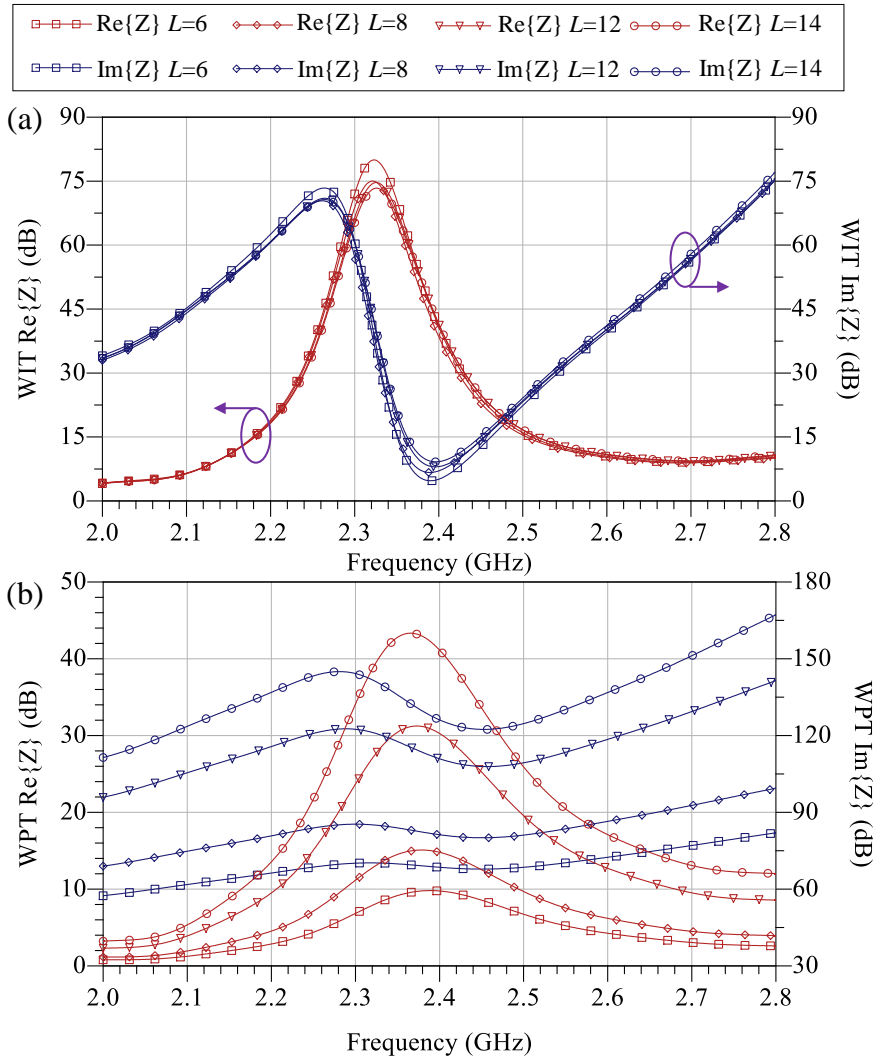

Fig. 5. Simulated input impedance of the antenna as a function of the tuning loop length $L_{\text {Loop }}$ (in $\mathrm{mm}$ ) at: (a) the $50 \Omega$-matched WIT port; (b) the complex $Z$-matched WPT port.

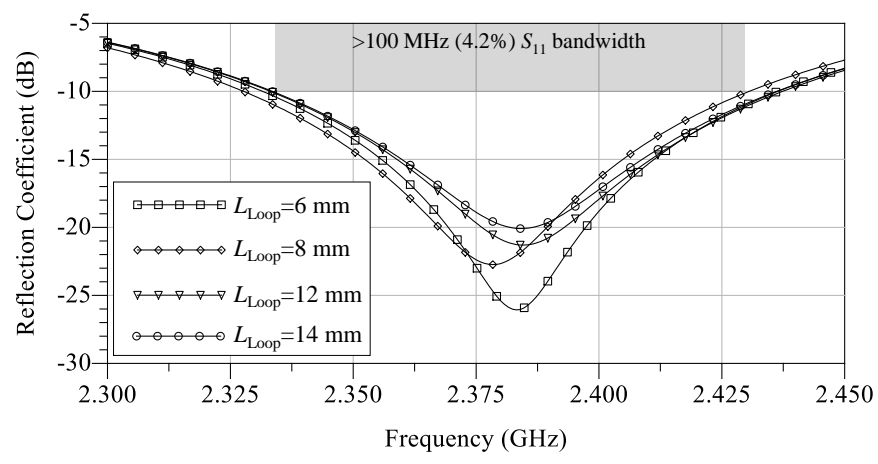

Fig. 6. Simulated $S_{11}$ bandwidth of the communications (WIT) ports for all $L_{\text {Loop }}$ values investigated.

and TM10 for the vertically and horizontally-polarized ports, respectively. Fig. 8 shows the simulated $E$-field distribution through the patch's substrate on the XY and YZ planes, for active excitations on ports 1 and 4, both generating a verticallypolarized main beam. From the $E$-field plot, it can be observed that the radiation mechanism is similar resulting in the same broadside off-body radiation pattern. As for the differentiallyfed complex- $Z$ port 4, in Fig. 8-c, a higher $E$-field distribution is observed around the feeding slot, similar to that observed in the proximity-coupled region of the microstrip line in Fig. 8-a, for the $50 \Omega$ port.

For the WPT port, the frequency of operation is expected to be slightly off the $\Re\{Z\}$ resonance peak, to achieve a complex 


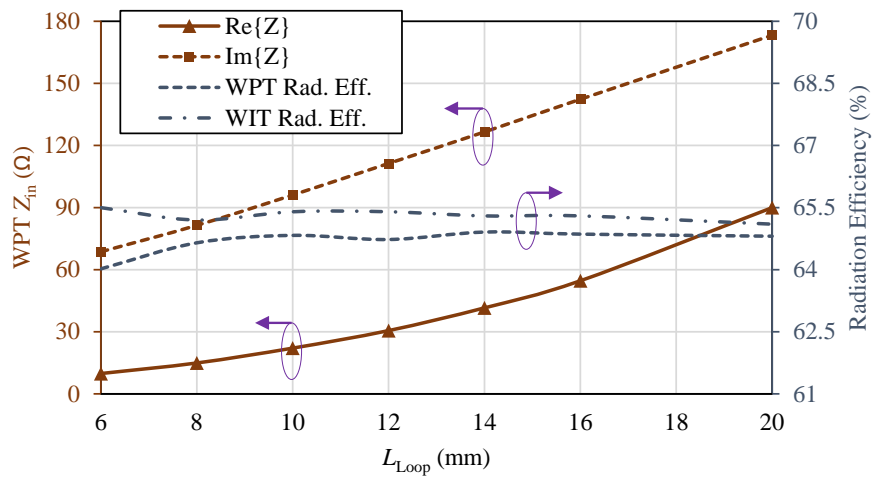

Fig. 7. Simulated input impedance of the rectenna at $2.4 \mathrm{GHz}$ as a function of $L_{\text {Loop }}$, and the radiation efficiency of the WPT/WIT ports.
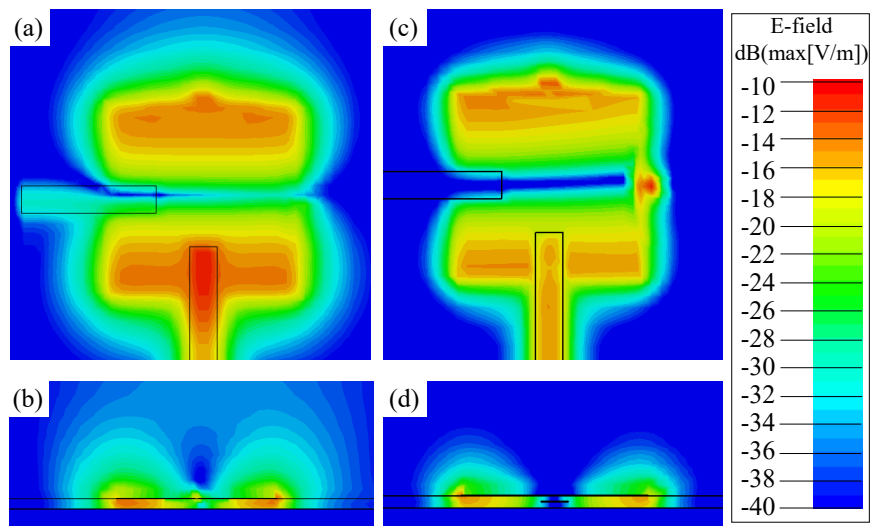

Fig. 8. Simulated $E$-field plots of the TM01 (vertically-polarized) mode of the proposed antenna from ports and 1 and 4. (a) XY plot of port 1 excitation; (b) YZ plot of port 1 excitation; (c) XY plot of port 4 excitation; (d) YZ plot of port 4 excitation.

conjugate match with the rectifier. Nevertheless, as observed in Fig. 7, both ports maintain a consistent radiation efficiency for varying $L_{\mathrm{Loop}}$, demonstrating that the impedance tunability does not affect the antenna's radiation properties.

As the proposed antenna is aimed at wearable applications, the Specific Absorption Rate (SAR) of the $2.4 \mathrm{GHz}$ transmitter port has been simulated using the Austinman human model [32]. Fig. 9 shows the antenna's position, with an $8 \mathrm{~mm}$ air gap between the antenna and the model. The SAR was simulated for a $0.5 \mathrm{~W}$ input at port-1, showing a peak SAR of 0.355 and $0.152 \mathrm{~W} / \mathrm{kg}$ when normalized to 1 and 10 gm tissue mass, respectively. For both cases the SAR is well bellow the $1.7 \mathrm{~W} / \mathrm{kg}$ limit of the IEEE C95.1 standard. Furthermore, compared to the previously developed wearable SWIPT antenna in [18], the proposed antenna achieves at least four-fold reduction in the SAR, owing to the full ground plane covering both the WPT and WIT ports resulting in better isolation from the body.

\section{WPT Rectenna and Rectifier Simulation}

The rectifier used in the implemented rectenna is a $2.4 \mathrm{GHz}$ voltage doubler based on the SMS7630-079LF Schottky diode. The rectifier is based on the same layout in [18]. The rectifier circuit has been analyzed using harmonic balance (HB)

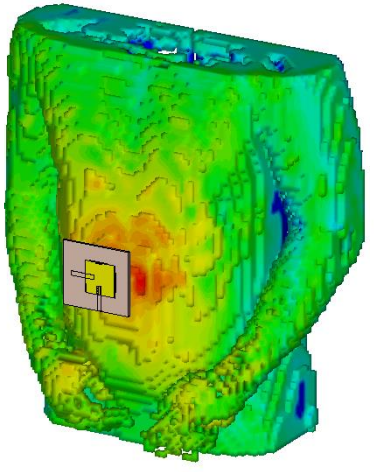

$0.5 \mathrm{~W}$ at $1 \mathrm{gm}: \mathrm{SAR}_{\text {peak }}=0.355$

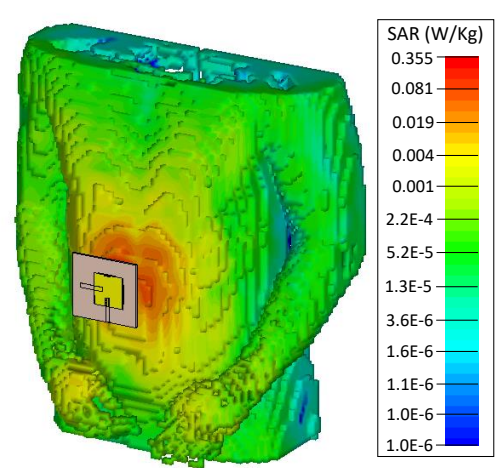

$0.5 \mathrm{~W}$ at $10 \mathrm{gm}: \mathrm{SAR}_{\text {peak }}=0.152$
Fig. 9. Simulated SAR of the antenna, normalized to 1 and 10 gm tissue mass, on an Austinman phantom for $0.5 \mathrm{~W}$ transmission power level at 2.4 $\mathrm{GHz}$.

simulation, in Keysight ADS, to identify the optimum input impedance for maximizing the power conversion efficiency (PCE); the extracted input impedance is then set as the target impedance of the antenna at both WPT ports. This antenna rectifier co-design approach not only eliminates the matching network, reducing the system's size, complexity, and cost, but it has also been demonstrated with best-in-class PCEs for PCB [33], flexible dipole [34], and fully-textile [18] rectennas.

The extracted optimum input impedance is $20+\mathrm{j} 130 \Omega$ at $2.4 \mathrm{GHz}$. As previously observed in Fig. 5, the complex input impedance can be tuned through the loop's length to achieve the desired input impedance. Based on the HB simulation, the optimum load impedance is $5.5 \mathrm{k} \Omega$ with a peak simulated PCE of $79 \%$. Owing to the WIT/WPT coupling, when the transceiver is actively absorbing power, the rectenna's ports are expected to maintain a $4 \mathrm{dBi}$ realized gain. The final antenna dimensions (in $\mathrm{mm}$ ) are chosen to be $W=L=50, L_{\mathrm{Loop}}=12$, and $H_{\text {Loop }}=30$. With a minimum feature size of $1 \mathrm{~mm}$, the antenna can be realized using most low-cost fabrication methods such as embroidery, conductive fabrics, or screen printing.

\section{Antenna Fabrication and Measurement}

\section{A. Textile Antenna/Rectenna Fabrication and Assembly}

The antenna has been fabricated using $0.1 \mathrm{~mm}$-thick conductive fabric (Metweave from $\mathrm{P} \& \mathrm{P}$ ) with a measured sheet resistance less than $50 \mathrm{~m} \Omega /$ square. The conductivity compares favorably against inkjet printed antennas on textiles [35] and is easier to apply to various substrates such as felt without the need for lossy printable dielectrics [5]. In addition, conductive fabric is more breathable and comfortable compared to copper films and polyimide copper laminates, mostly used for wearable antennas beyond $20 \mathrm{GHz}$ [9], [36], [37].

The antenna's traces were cut using an Epilog Mini 24 laser cutter with a $60 \mathrm{~W} \mathrm{CO}_{2}$ laser. Copper-clad Kapton polyimide has been used for the rectifier as in [3]. As polyimide (Kapton) can withstand temperatures up to $400^{\circ} \mathrm{C}$, the diodes can be soldered directly and encapsulated using vacuum-formed polyimide for improved mechanical reliability and to withstand machine washing as demonstrated in [38]. Fig. 10-a shows a photograph of the fabricated antenna prototype. 


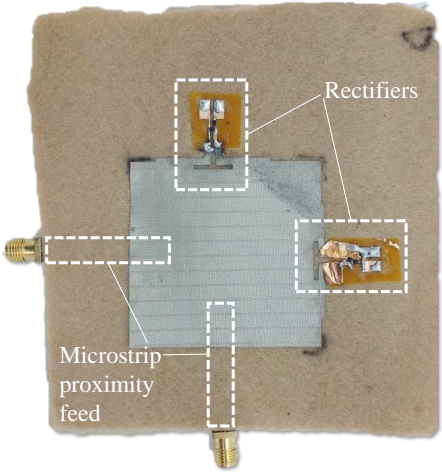

(a)

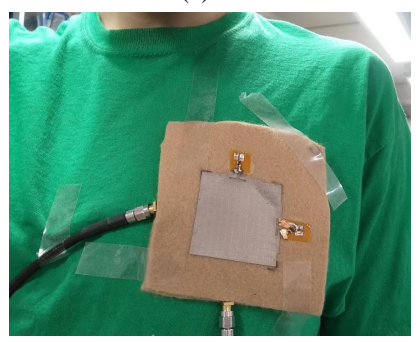

(c)

Fig. 10. Photographs of the proposed antenna and the on-body test setups: (a) the fabricated prototype; (b) two-port measurements on-wrist; (c) on-chest measurements; (d) on-wrist measurement in proximity with a smart watch.

\section{B. Two-Port Antenna Measurements}

The antenna's s-parameters were measured using a ZVB4 vector network analyzer (VNA) with a standard TOSM calibration. The two-port s-parameters were measured for the $50 \Omega$ matched ports 1 and 2, i.e. the WIT ports. Fig. 11 shows the simulated and measured s-parameters of the proposed antenna, prior to loading the rectifier with a resistive load, or exposure to bending or human proximity effects. The close agreement between the simulated and measured results demonstrates the high isolation achieved by the proposed feed, as well as the unaltered input impedance matching for the WIT ports. The discrepancy observed between the reflection coefficients $S_{11}$ and $S_{22}$, despite the antenna's symmetrical design, is attributed to variations in the SMA connector mounting and the manual assembly of the antenna, and has previously been observed on dual-port textile antennas [39]. The envelope correlation coefficient (ECC) calculated from the measured s-parameters and the simulated far-field patterns, shown in Fig. 11, demonstrate the antenna's suitability for MIMO applications.

The antenna was then measured on a user's wrist and chest, as shown in Fig. 10-b and c. On-wrist, the $S_{11}$ was measured for bending on both the $E$-plane (Wrist-A) and $H$ plane (Wrist-B). The measured loaded $S_{11}$ is shown in Fig. 12, where it can be observed that the antenna does not detune in proximity with the body, or when bent around the wrist.

From Fig. 12, it can also be observed that the antenna maintains at least $15 \mathrm{~dB}$ port isolation between both WIT ports, showing its suitability for MIMO and full-duplex applications with favorable performance compared to recent textile implementations [13], [14]. To illustrate, previously reported dual-polarized microstrip patch antennas, [11], [13],

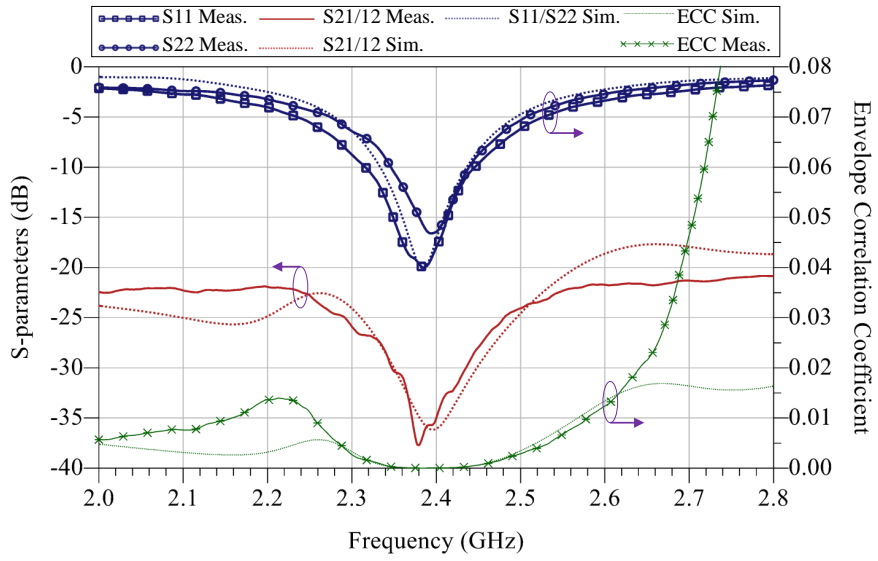

Fig. 11. Simulated and measured s-parameters of the antenna's WIT ports, and the calculated MIMO ECC.

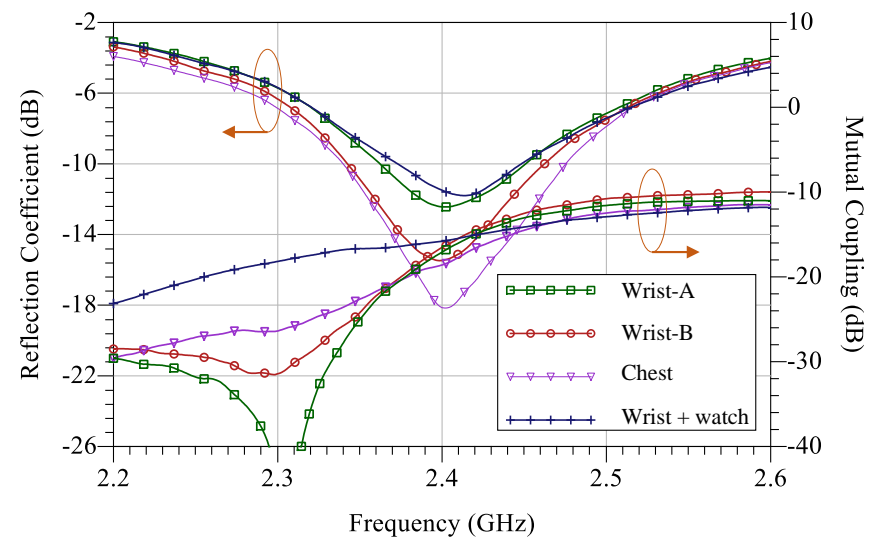

Fig. 12. Measured s-parameters of the WIT ports, after connecting the rectifier, while bending around different on-body positions.

[14], have shown an increase in the mutual coupling between the ports when the antennas were measured on-body and under bending. To explain, bending and twisting the microstrip feed line alters the exact feeding point of the patch and results in additional coupling between the orthogonal microstrip lines. The high isolation maintained by the proposed antenna, compared to previous designs, is attributed to the hybrid inductive/proximity-coupled feed.

In a real use-case, the antenna may be operating near other wearable devices and metallic objects, such as smart watches, which could potentially alter the $S_{11}$ by detuning the antenna or reduce the port isolation. Therefore, the antenna's $S_{11}$ was measured in proximity with a smart watch (approximately 2 $\mathrm{cm}$ clearance); the watch overlaps the felt substrate as well as the textile ground plane as shown in Fig. 10-d. The measured s-parameters in proximity with the watch (in Fig. 12) show that while port-1 whose $E$-plane intersects with the watch observes an increase in the $S_{11}$, the antenna maintains its $S_{11}<-10 \mathrm{~dB}$ at $2.4 \mathrm{GHz}$ and the ports' isolation remains unaffected. However, as the on-wrist bending in proximity with a metal watch case (Fig. 10-d) exhibits the highest variation in the $S_{11}$ response, future wearable antenna designs should be characterized in proximity with other wearable devices and not just tissue and clothing. Should the textile 


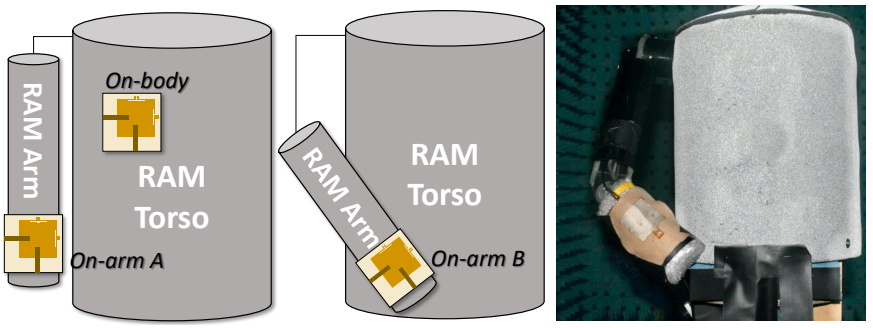

Fig. 13. Layout of the antenna's measurement setup on the RAM body phantom; photograph shows the antenna on-arm in position B in an anechoic chamber.

antenna overlap the metallic wearables or accessories, the $S_{11}$ response will be maintained due to the full textile ground plane backing.

\section{Radiation Properties Measurements}

The antenna's 3D polarimetric radiation patterns of the antenna were measured in an anechoic chamber on a solid radiation absorbing material (RAM) body phantom to measure its 3D directivity and total efficiency. The phantom's RAM composition enables the emulation of body-shadowing and inbody losses [40]. The antenna was measured on the phantom's body and arm. On-arm, the antenna was measured on the body's side as well as positioned diagonally in front of the body, as shown in Fig. 13. By bending and adhering the antenna to conform to the "body" parts, the impact of bending on the antenna's radiation properties is included in the measured patterns. The radiation properties were measured with both rectifiers connected on ports 3 and 4, to account for the impact of simultaneous WPT and WIT. The simulated and measured normalized radiation patterns are shown in Fig. 14.

In free space, the antenna's simulated patterns exhibited over $25 \mathrm{~dB}$ co/cross-polarization isolation on the principal $E$ and $H$-planes. However, when the antenna is placed on the body, the cross-polarization "null" is filled by the reflected waves off-body, resulting in a higher cross-polarized component with 12-20 dB polarization purity, as summarized in Table I. This has previously been observed in a range of off-body textile antenna designs when the radiation patterns were measured on a phantom [5], [13]. Moreover, polarization purity is not of paramount significance in a mobile wearable environment [11], [13], where the main purpose of a dualpolarized antenna is overcoming the polarization misalignment losses in off-body links [25]. The variation observed between the simulated and measured radiation patterns is due to the differences between the Austinman model used in CST and the RAM phantom used in measurements.

It can also be observed that for the two on-arm positions, the polarization on port- 2 maintains $5-13 \mathrm{~dB}$ co/cross isolation. It was previously observed that the co/cross-polarization isolation could degrade to under $5 \mathrm{~dB}$ on a user's wrist [16]. Moreover, the cross-polarized pattern exhibited asymmetry between both ports of a supposedly symmetric antenna design [16]. These effects are attributed to the off-body reflections, where the antenna's pattern on each of the orthogonal ports will depend on the port's interaction with the body based on the arm/wrist position. Thus, in a real-world use-case, the polarization purity of an off-body antenna will depend on the antenna's position [25], as demonstrated by the variation in the measured radiation patterns for the two on-arm positions in Fig. 14. It is difficult to assess the polarization purity of other reported wearable MIMO/full-duplex antenna designs, such as [13], [14], [16], as the polarized patterns were only reported in space or for a single on-wrist orientation [16].

The total (radiation+matching) efficiency of the antenna has been evaluated with respect to a reference low-loss monopole ( $\lambda / 4$ monopole over a large ground plane) using the measured total radiated power from both antennas. The measured total efficiency is given by

$$
\eta_{\text {Tot. }}=\frac{\oiint_{S}\left|E_{\text {Textile }}\right|^{2} d S}{\oiint_{S}\left|E_{\text {Monopole }}\right|^{2} d S},
$$

where $E$ is the total measured radiated electric field from the (irrespective of polarization) antenna-under-test, in the same test conditions, and $S$ represents the surface surrounding each antenna [41]. The measured efficiencies are given in Table I ( $\pm 5 \%$ based on [41]). In CST, the antenna's simulated efficiency on the Austinman phantom is $67 \%$. Previous studies have also reported a higher measured efficiency compared to the full-wave simulations for textile antennas, which is typically attributed to an overestimation of the $\tan \delta$ [13], in this case introduced by the additional air gaps in the multilayered fabric substrate.

The measured parameters of the communications antenna ports (port 1 and 2) are summarized in Table I. To explain, as the antenna-under-test is bent around the user/phantom's arm and body, as well as rotated for the 3D pattern measurements, additional variations to the measured $S_{11}$ can reduce the accepted power and subsequently the measured total efficiency. As for the on-arm setup, the alignment of the arm with the body will change how the radiated waves diffract and reflect off-body. Therefore, for a straight and a bent arm (configurations $\mathrm{A}$ and $\mathrm{B}$ on-arm), the total efficiency is higher on port-1 and port-2, respectively. This highlights the importance of dual-polarization and multi-port antennas in body-centric communications. For example, the $1 \mathrm{~dB}$ higher total radiated power from port-1 compared to port-2 in configuration-A shows that should the proposed antenna be used for off-body MIMO communications, the link-budget could be improved due to the higher total radiated power for a certain port.

\section{Rectenna Simulation and Measurements}

The first step in evaluating the rectenna is the input impedance measurements of the rectifier's differential port. A balanced coaxial jig has been use to measure the input impedance using a two-port imbalanced VNA, based on the method described in [42] previously used to characterize textile-based RFID and discrete rectennas with high accuracy [18], [38]. The impedance was measured under two conditions, with an open termination on the communications ports, as well as terminated using SMA $50 \Omega$ loads to emulate the active 

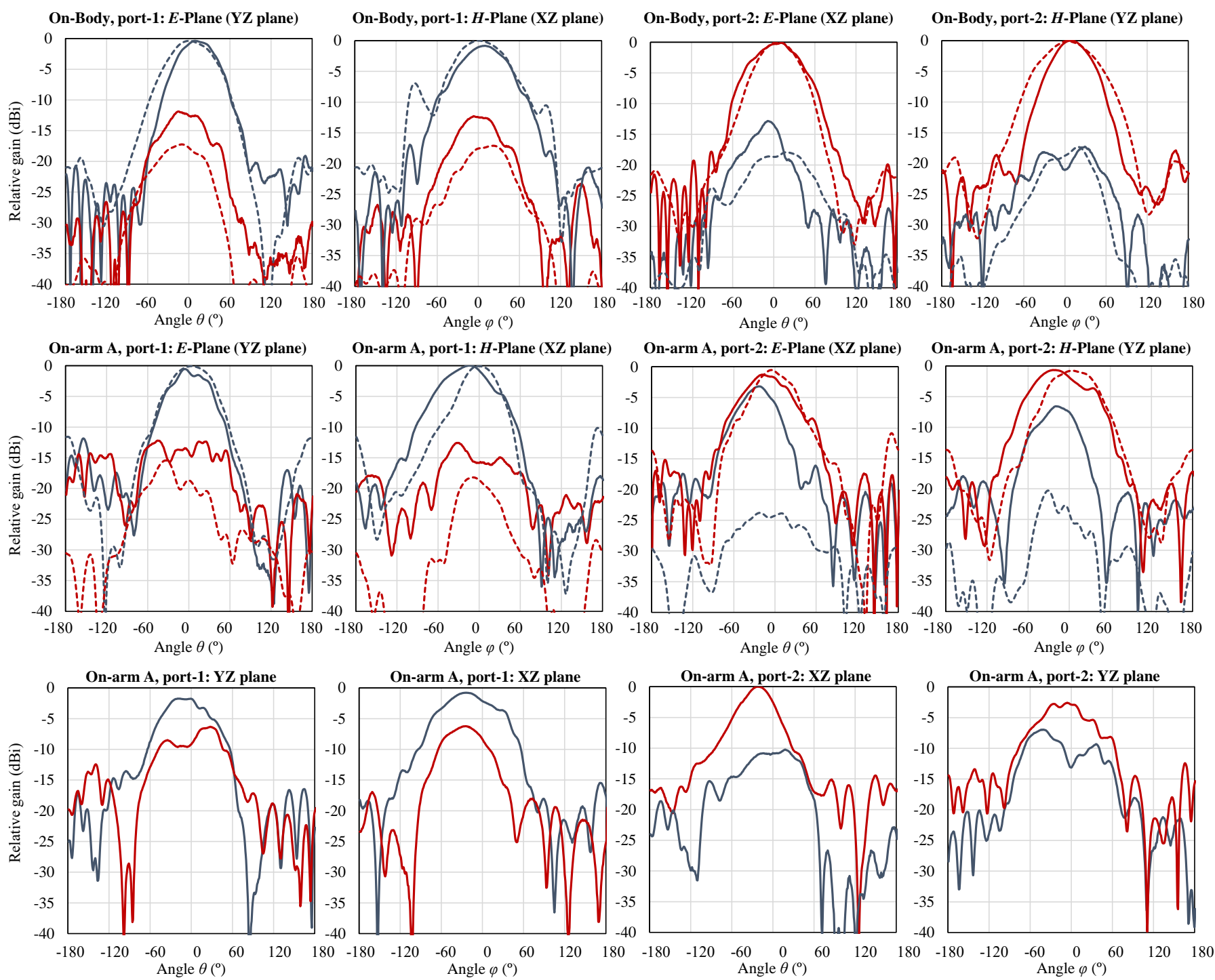

Fig. 14. Simulated (dashed) and measured (solid) vertically (blue) and horizontally (red) polarized normalized radiation patterns of the antenna, measured across both ports for different on-phantom positions.

TABLE I

Measured antenna's COMms. PERformance at $2.4 \mathrm{GHz}$.

\begin{tabular}{|l|l|l|l|l|l|l|}
\hline & $\begin{array}{l}\text { Port-1 } \\
\text { body }\end{array}$ & $\begin{array}{l}\text { Port-2 } \\
\text { body }\end{array}$ & $\begin{array}{l}\text { Port-1 } \\
\text { arm-A }\end{array}$ & $\begin{array}{l}\text { Port-2 } \\
\text { arm-B }\end{array}$ & $\begin{array}{l}\text { Port-1 } \\
\text { arm-A }\end{array}$ & $\begin{array}{l}\text { Port-2 } \\
\text { arm-B }\end{array}$ \\
\hline \hline Tot. $\eta$ & $73 \%$ & $76.4 \%$ & $83 \%$ & $71 \%$ & $88.4 \%$ & $70 \%$ \\
\hline $\begin{array}{l}D \\
(\mathrm{dBi})\end{array}$ & 10.7 & 10.8 & 10.1 & 10.0 & 9.5 & 10.0 \\
\hline $\begin{array}{l}\text { Rlz. } G \\
(\mathrm{dBi})\end{array}$ & 9.3 & 9.6 & 9.3 & 8.5 & 9.0 & 8.4 \\
\hline $\begin{array}{l}S_{21} \\
(\mathrm{~dB})\end{array}$ & -18.6 & NA & -17.0 & NA & -16.5 & NA \\
\hline $\begin{array}{l}\mathrm{Co} / \mathrm{X}- \\
\text { pol. } \\
(\mathrm{dB})\end{array}$ & 12.7 & 20.9 & 13.9 & 7.4 & 6.5 & 11.8 \\
\hline
\end{tabular}

NA: not-applicable due to ports reciprocity; Co/X-pol: polarization isolation.

transceivers. Fig. 15 shows the simulated and measured input impedance across both WPT ports of the antenna. The target rectifier impedance $Z_{\text {Rect. }}$ is included on the same plot.
While the antenna is symmetrical, the measured $Z_{\text {in }}$ shows variations over both ports. This is attributed to the measurement uncertainty introduced by the landing position of the coaxial jig on the rectifier's feed, along with fabrication tolerances. To explain, placing the coaxial jig's tips further away from the loop increases the observed $Z_{\text {in }}$ (an impedance transformer effect [3]). When the antenna's WIT ports were not terminated (Fig. 15-b), an additional resonance is observed around $2.8 \mathrm{GHz}$ which is attributed to the standing waves in the open-ended proximity-coupled lines, acting as resonant stubs. However, the impedance remains mostly unchanged at $2.4 \mathrm{GHz}$ indicating an unaffected WPT performance regardless of the termination on the WIT ports

Although co-designed complex- $Z$ rectennas have been widely demonstrated [33], including on textiles for SWIPT [18], their impedance has not been measured in human proximity. Therefore, the input impedance of the proposed antenna was measured on the user's body, demonstrating (in Fig. 15a) that the proposed antenna's WPT port does not detune in 

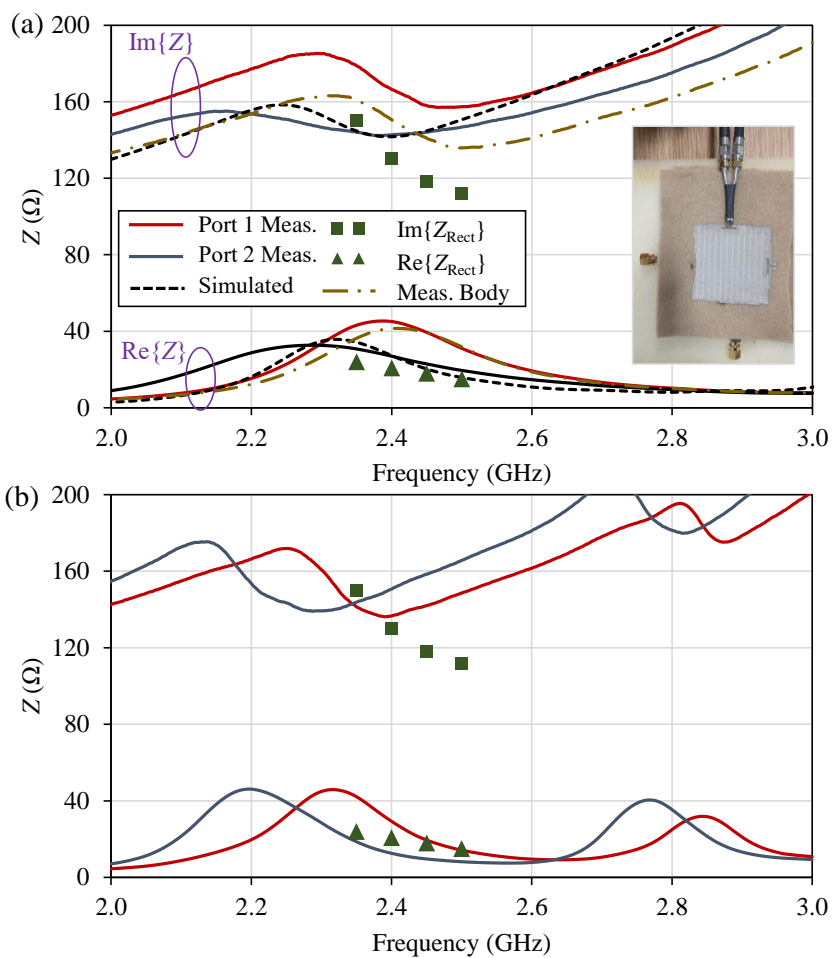

Fig. 15. Simulated and measured input impedance of the rectifier ports: (a) with a $50 \Omega$ termination on the WIT ports; (b) with open-ended SMA's on the WIT ports; inset shows the measurement setup

human proximity. This is attributed to the full ground plane backing providing high isolation from the body.

As the rectenna has a complex $Z$, it is not possible to directly characterize the rectifier using a signal generator. The DC voltage $V_{\mathrm{DC}}$ has been measured using an oscilloscope across a $Z_{\text {load }}=5.5 \mathrm{k} \Omega$. A $1 \mathrm{~W}$ power amplifier has been connected to a transmitting $9 \mathrm{dBi}$ patch antenna for generating $0-40 \mathrm{dBm}$ equivalent isotropic radiated power (EIRP). The rectenna was positioned at $50 \mathrm{~cm}$ from the transmitter (satisfying the Fraunhofer far-field distance at $2.4 \mathrm{GHz}$ [24]). The $S_{21}$ between the transmitting VNA continuous wave (CW) port, and the WIT port on the antenna/rectenna was used to estimate the incident power density $S$, and subsequently calculate the PCE as the ratio of the DC power across the load to the RF power received by the antenna, and is given by

$$
\mathrm{PCE}=\frac{V_{\mathrm{DC}}^{2}}{Z_{\text {load }} A_{\mathrm{eff}} S} .
$$

For the effective area calculation, the simulated realized gain $G_{\mathrm{RX}}=4 \mathrm{dBi}$, inclusive of the mutual coupling losses with the WIT ports, has been used to calculate $A_{\text {eff }}$. Fig. 16 shows the simulated and measured PCE of the rectenna.

The PCE of the rectenna has also been measured with the antenna positioned on-body, with the same distance between the transmitter and the rectenna [24]. As the human body temperature may influence the PCE due to the diode's temperature-dependence, the temperature of the rectenna was measured using an infrared thermometer to be around $27^{\circ} \mathrm{C}$, where the room temperature was $25^{\circ} \mathrm{C}$ and the skin tempera-

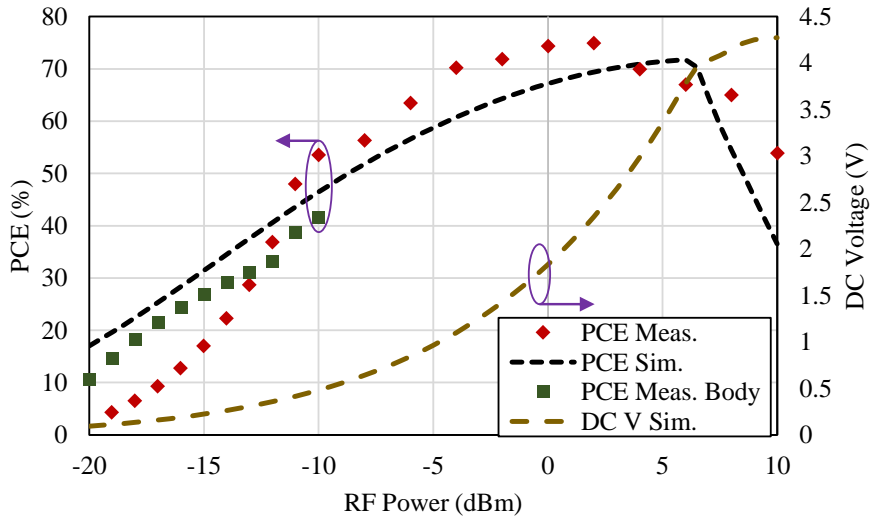

Fig. 16. Simulated and measured PCE of the proposed rectenna at $2.4 \mathrm{GHz}$ for a $5.5 \mathrm{k} \Omega$ load.

ture was $34^{\circ} \mathrm{C}$. Fig. 16 shows the measured PCE of the antenna on the body.

The main discrepancy observed between the simulated and measured PCE in Fig. 16 is observed under $-10 \mathrm{dBm}$. This is attributed to the non-linear coupling between the rectifier and the WIT ports, where the leakage in the $50 \Omega$ terminations prohibits the rectifier from receiving a sufficient power level to achieve its simulated PCE, which was simulated based on an ideal source with a matched $Z_{\text {in }}$. Moreover, the variations between the on-body and in-space performance are attributed to errors in estimating the incident RF power level during wireless testing, which typically introduces a $\pm 1 \mathrm{~dB}$ error [5]. Nevertheless, the PCE $>50 \%$ region from -10 to $10 \mathrm{dBm}$ is in line with recent wearable textile rectenna implementations [6]. For instance, the textile rectifier in [6], operating at $2.4 \mathrm{GHz}$, achieves a $70 \%$ PCE at $10 \mathrm{dBm}$. Above $-5 \mathrm{dBm}$, the higher measured PCE compared to simulation could be attributed to overestimating the antenna's losses, i.e. underestimating the gain, resulting in a higher $G_{\mathrm{RX}}$ increasing the harvested power from the same $S$.

As the rectenna directly matches the rectifier and adopts a differential feed, directly measuring the RF gain patterns is challenging. Alternatively, the DC output patterns can be used to evaluate the rectenna's power harvesting angular patterns [3], [43]. In [43], it was found that a rectifier comparable in size with the antenna will result in the power harvesting pattern deviating from the antenna's RF gain pattern at $2.45 \mathrm{GHz}$. However, for the very compact rectifier in [3] the DC power patterns were found to closely match the CST-simulated gain. Therefore, practical measurements of the rectenna's DC output as a function of the angle of incidence are essential to characterize its power harvesting performance.

The wireless power source has been moved to fixed angular points around the textile rectenna. In the broadside direction ( $\theta=0$ in Fig. 17), the received RF power is estimated as $-10 \mathrm{dBm}$, based on the measured power level at the copolarized communications port on the same antenna. Fig. 17 shows the CST-simulated gain of the patch at the WPT port, and the measured DC output for varying angles of incidence around the rectenna. The measured DC output closely matches the simulated gain pattern, showing that the rectenna maintains 


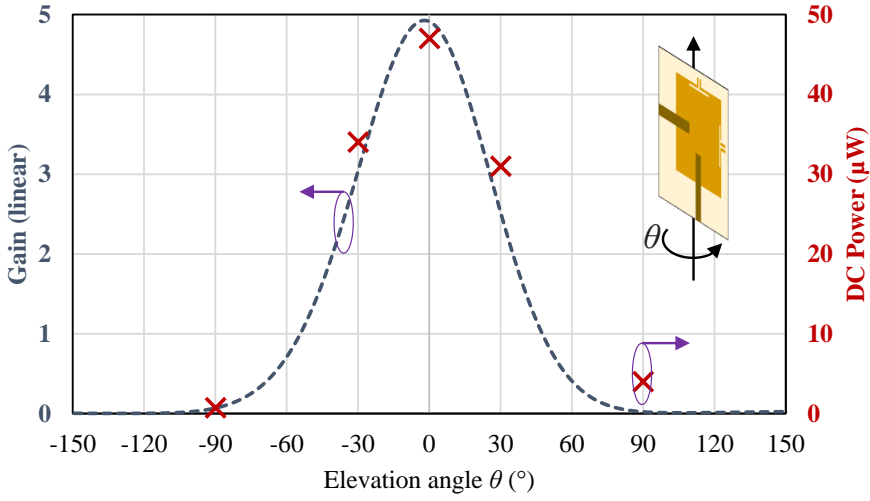

Fig. 17. Simulated gain (continuous line) and the measured DC power (discrete points) around the antenna for a $-10 \mathrm{dBm}$ input at $\theta=0^{\circ}$.

a broadside harvesting aperture. The wider beamwidth of the rectenna, as well as the higher DC power output observed behind the antenna can be attributed to multi-path reflections in the indoor test environment.

Following the power harvesting pattern investigation, the power splitting between both the vertically and horizontallypolarized ports is investigated, for a $45^{\circ}$ misaligned incident wave. At $0 \mathrm{dBm}$, where the rectenna's PCE peaks, the PCE of each individual port drops by $20 \%$, owing to the lower power received. Considering previously reported dual-polarization rectennas, directly combining the harvested power would result in an approximately $30 \%$ reduction in the harvested DC power compared to single polarization harvesting [44]. More complex power combining circuits have been proposed for scenarios where the power splitting is uneven between the ports [45], which can be applied to a dual-polarized rectenna with DC combining. Moreover, the use of hybrid RF and DC combining can reduce the dual-polarization loss to under 5\% [46]. However, such an approach requires a separate matching network due to the $50 \Omega$ matching of the hybrid coupler, increasing the complexity of the implementation which is not compatible with the proposed rectenna.

\section{E. Large-Signal Port Isolation Measurements}

As the rectifier is a non-linear component, the mutual coupling between the WIT and WPT ports will be a function of the power level at which information is transmitted or received. Furthermore, as it was observed in simulation that the presence of the rectifier and its inductive feed can result in variations in the mutual coupling between the dual-polarized communication ports, it is essential to characterize the largesignal coupling between the antenna's four ports.

As shown in the inset in Fig. 18, the parasitic power leakage into the rectifier from the WIT ports is estimated based on the rectified DC voltage, measured using an oscilloscope. The open circuit voltage was used to estimate the RF power absorbed by the rectifier based on a look-up table, from the nonlinear HB simulations of the rectifier. This has been performed for the co-polarized WIT/WPT ports $\left(S_{32}\right)$, the orthogonallypolarized WIT/WPT ports $\left(S_{31}\right)$, as well as for RF power being transmitted from both port 1 and 2 simultaneously $\left(S_{31}+S_{32}\right)$.

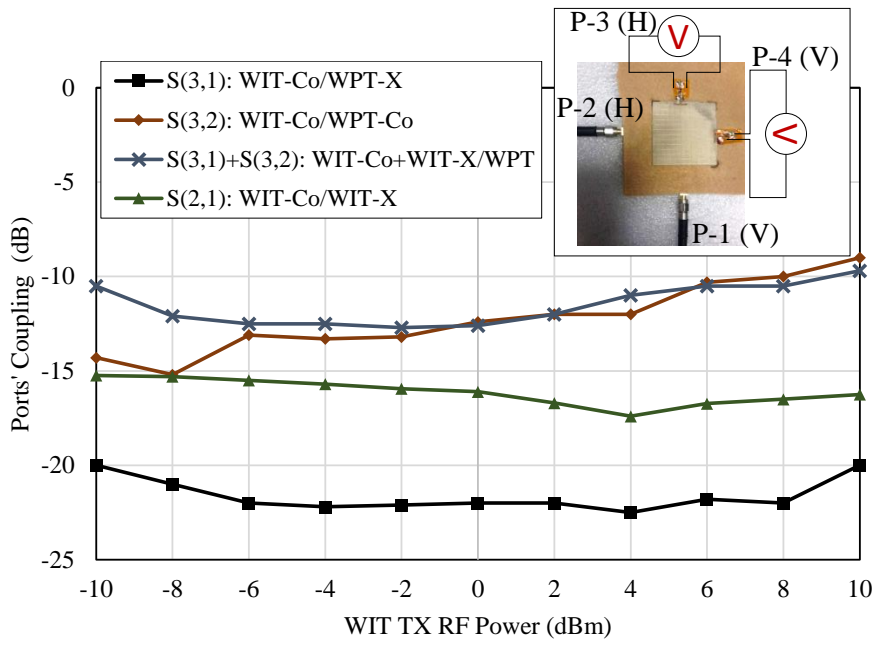

Fig. 18. Measured large-signal mutual coupling between the antenna's four ports.

As for the WIT/WIT coupling $S_{21}$, this has been measured directly using the VNA. The VNA was used as a power source in this setup with a varying input power level between -10 to $10 \mathrm{dBm}$. Fig. 18 shows the measured mutual coupling between the ports of the antenna.

Intuitively, as simulated in Section II-B, and as previously observed in [24], the highest isolation is observed between the cross-polarized $50 \Omega$ WIT and complex- $Z$ WPT ports $\left(S_{31}\right)$. On the other hand, the coupling between the co-polarized WIT/WPT ports $\left(S_{32}\right)$ is the highest and approaches $9.5 \mathrm{~dB}$ for a $10 \mathrm{dBm}$ input. This was found to increase with the RF power level due to the non-linearity of the diodes and their improved matching at higher power levels. The WIT/WIT coupling $\left(S_{21}\right)$ is expected to be power-independent in a standard dual-port passive antenna. Nevertheless, the presence of the non-linear rectifiers results in a power dependence in the $S_{21}$ measured by the VNA. However, for all power levels investigated, the WIT/WIT port isolation is over $15 \mathrm{~dB}$, showing the antenna's suitability for MIMO and full-duplex applications.

\section{F. Comparison with Previous Work}

The proposed antenna is distinguished from recent SWIPT antennas/rectennas by being the only antenna for dualpolarized SWIPT [18], [20], [22], in addition to maintaining lower complexity and more uniform radiation properties compared to a recent dual-band SWIPT microstrip rectenna [18]. In [22], a matching network is required to match the hybrid coupler to the rectifier, increasing the complexity of the implementation compared to the proposed antenna. As for [20], both polarizations of the sub-1 GHz harvest power, one through a discrete rectifier and the other through a fully-integrated charge pump inside the RFID chip. However, only the RFID chip port can communicate via backscattering modulation, limiting the antenna's applicability to single polarization passive communication. Therefore, the proposed antenna is the only reported implementation, amongst both wearable and non-wearable antennas, capable of simultaneously receiving information and power for two orthogonal polarizations. 
TABLE II

COMPARISON OF THE PROPOSED ANTENNA WITH RECENT MULTI-PORT TEXTILE AND WEARABLE ANTENNAS.

\begin{tabular}{|c|c|c|c|c|c|c|c|c|c|}
\hline & Ports & Application & $\begin{array}{l}\text { Frequency } \\
(\mathrm{GHz})\end{array}$ & $\begin{array}{l}\text { Comms. } \\
\text { Gain (dBi) }\end{array}$ & $\begin{array}{l}\text { Total WIT } \\
\text { Efficiency } \\
\end{array}$ & $\begin{array}{ll}\begin{array}{l}\text { Port } \\
(\mathrm{dB})\end{array} & \text { iso. } \\
\end{array}$ & $\begin{array}{l}\text { Co/X-pol } \\
\text { iso. }(\mathrm{dB})\end{array}$ & $\begin{array}{l}1 \mathrm{gm} \text { SAR } \\
(\mathrm{W} / \mathrm{kg})\end{array}$ & $\begin{array}{ll}\text { Peak } & \text { WPT } \\
\text { PCE } & \\
\end{array}$ \\
\hline This work & 4 & SWIPT & 2.4 & $\begin{array}{l}\text { WIT: } \\
8.4-9.6 \\
\text { WPT: } \\
2.5-4\end{array}$ & $70-88 \%$ & $\begin{array}{l}\text { WIT/WIT: } \\
16-35 \mathrm{~dB} \\
\text { WPT/WIT: } \\
4-10 \mathrm{~dB}\end{array}$ & $6-20 \mathrm{~dB}$ & 0.355 & $\begin{array}{l}74 \% \quad \pm 5 \% \\
\text { at } \\
2 \mathrm{dBm}\end{array}$ \\
\hline [3] & 2 & WPT & 0.83 & NA & NA & 13 & NR & $\begin{array}{l}0.876^{\dagger} ; \\
4.4^{\ddagger}\end{array}$ & $\begin{array}{l}58 \% \text { at } \\
-10 \mathrm{dBm}\end{array}$ \\
\hline [11] & 2 & WIT & 5.8 & $4-7$ & $85-90 \% *$ & 25 & NR & 0.29 & NA \\
\hline [13] & 2 & WIT & 2.4 & 7.5 & $90 \% *$ & 16 & 12 & $0.12^{\dagger} ; 0.6^{\ddagger}$ & NA \\
\hline [14] & 2 & WIT & 2.4 & 1.7 & $29 \%$ & $12-17 \mathrm{~dB}$ & $\approx 10^{* *}$ & $0.44^{\ddagger}$ & NA \\
\hline [15] & 2 & WIT & $2.4 / 5$ & 2.0 & $50 \%$ & 20 & $\approx 5-10^{* *}$ & 0.056 & NA \\
\hline$[16]$ & 2 & WIT & 2.4 & 4.2 & $60.7 \%$ & 15 & $\approx 7-10^{* *}$ & 0.55 & NA \\
\hline [18] & 2 & SWIPT & $0.83 / 2.4$ & 7.2 & $63-66 \%$ & $25-30$ & $\mathrm{NA}$ & 1.9 & $\begin{array}{lr}62 \% & \text { at } \\
0.8 \mu \mathrm{W} / \mathrm{cm}^{2}\end{array}$ \\
\hline
\end{tabular}

NA: not applicable; NR: not reported; iso: isolation; ; ${ }^{*}$ simulated result; ${ }^{* *}$ estimated from the graph; ${ }^{\dagger} 0.1 \mathrm{~W}$ input; ${ }^{\ddagger}$ calculated for $0.5 \mathrm{~W}$ input.

The proposed antenna/rectenna is compared in Table II to recent multi-port wearable textile antennas. From the measured radiation efficiency and gain, in addition to the ports' isolation, it can be observed that the proposed antenna achieves superior communications performance, despite having two ports connected to "always on" rectifiers for simultaneous power harvesting. In addition, owing to the full ground plane backing, a higher rectenna gain is maintained compared to [24], where the rectenna's port and radiating element lack any isolation from the human body. This also contributes to a very low SAR of $0.355 \mathrm{~W} / \mathrm{kg}$ for $0.5 \mathrm{~W}$ power input at either of the WIT ports. While [13] and [11] report higher radiation efficiencies (exclusive of mismatch), the reported efficiencies are based on simulated results only, whereas the proposed antenna was measured in direct contact with a lossy phantom, resulting in no clearance between the antenna and the tissue-mimicing RAM. Furthermore, while [15] achieves high $(20 \mathrm{~dB})$ isolation between the dual MIMO ports, two antenna elements are used with a $0.08 \lambda$ spacing, increasing the size and complexity of the implementation.

\section{CONCLUSION}

In this paper, a dual-polarized four-port fully-textile antenna has been proposed for SWIPT applications in MIMO and fullduplex BANs, the first antenna designed for simultaneously harvesting power and communicating over two orthogonal polarizations. Owing to the hybrid feeding approach using capacitive proximity coupling for the $50 \Omega$ communications ports and differential inductive loop feed for the WPT ports, the antenna maintains at least $10 \mathrm{~dB}$ port isolation for all ports in both small and large-signal measurements, and up to $35 \mathrm{~dB}$ isolation between the communications ports. The antenna achieves $100 \mathrm{MHz}$ bandwidth around $2.4 \mathrm{GHz}$ and maintains an $S_{11}<-10 \mathrm{~dB}$ while conforming to different body parts as well as when used in proximity with other wearables. Experimentally, the antenna maintains at at least $8.4 \mathrm{dBi}$ measured realized gain and $70 \%$ total WIT efficiency on a body phantom. The rectenna maintains a peak PCE of over $70 \%( \pm 5 \%)$ with a simulated gain around $4 \mathrm{dBi}$, inclusive of mutual coupling effects with the WIT ports. Furthermore, the radiation patterns have been measured in the most extensive on-phantom test setups compared to previous work. The rectifier is demonstrated with a PCE in line with recent WPT-only rectennas, in addition to having a feed geometry which could be tuned to match different input impedances based on the power level or diode model. For off-body communications, the proposed antenna compares favourably with reported dual-polarization wearable antennas showing that the addition of the rectifier ports does not degrade the antenna's performance in terms of port isolation, off-body gain, efficiency, and polarization-purity.

\section{ACKNOWLEDGMENT}

The authors would like to thank the Communication Systems and Networks Group at the University of Bristol for providing access to the antenna measurement facilities.

Datasets supporting this work can be accessed from the DOI: $10.5258 /$ SOTON/D1899

\section{REFERENCES}

[1] T. D. P. Perera, D. N. K. Jayakody, S. K. Sharma, S. Chatzinotas, and J. Li, "Simultaneous Wireless Information and Power Transfer (SWIPT): Recent Advances and Future Challenges," IEEE Communication Surveys and Tutorials, vol. 20, 1, pp. $264-302,2018$.

[2] Z. Hu, C. Yuan, and F. Gao, "Maximizing harvested energy for fullduplex swipt system with power splitting," IEEE Access, vol. 5, pp. 24975-24 987, 2017.

[3] M. Wagih, A. S. Weddell, and S. Beeby, "Omnidirectional DualPolarized Low-Profile Textile Rectenna with over 50\% Efficiency for Sub- $\mu \mathrm{W} / \mathrm{cm}^{2}$ Wearable Power Harvesting," IEEE Trans. Antennas Propag., vol. Early Access, 2020.

[4] G. Monti, L. Corchia, and L. Tarricone, "UHF Wearable Rectenna on Textile Materials,” IEEE Trans. Antennas. Propag., vol. 61, 7, pp. 3869 - 3873, 2013.

[5] S.-E. Adami, P. Proynov, G. S. Hilton, G. Yang, C. Zhang, D. Zhu, Y. Li, S. P. Beeby, I. J. Craddock, and B. H. Stark, "A Flexible 2.45GHz Power Harvesting Wristband With Net System Output From -24.3 $\mathrm{dBm}$ of RF Power," IEEE Trans. Microw. Theory Techn., vol. 66 no. 1, pp. 380-395, 2018.

[6] D. Vital, S. Bhardwaj, and J. L. Volakis, "Textile Based Large Area RF-Power Harvesting System for Wearable Applications," IEEE Trans. Antennas Propag., vol. 68, no. 3, pp. 2323 - 2331, 2019.

[7] J. A. Estrada, E. Kwiatkowski, A. López-Yela, M. Borgoñós-García, D. Segovia-Vargas, T. Barton, and Z. Popović, "An RF-Harvesting Tightly-Coupled Rectenna Array Tee-Shirt with Greater than Octave Bandwidth," IEEE Trans. Microw. Theory Techniq., vol. 68 no. 9, pp. $3908-3919,2020$. 
[8] M. Wagih, N. Hillier, S. Yong, A. S. Weddell, and S. Beeby, "Rfpowered wearable energy harvesting and storage module based on etextile coplanar waveguide rectenna and supercapacitor," IEEE Open Journal of Antennas and Propagation, vol. 2, pp. 302 - 314, 2021.

[9] M. Wagih, G. S. Hilton, A. S. Weddell, and S. Beeby, "Broadband Millimetre-Wave Textile-based Flexible Rectenna for Wearable Energy Harvesting ," IEEE Trans. Microw Theory Techn, vol. 68 no. 11, pp. 4960 - 4972, 2020.

[10] M. Wagih, O. Cetinkaya, B. Zaghari, A. S. Weddell, and S. Beeby, "Real-World Performance of Sub-1 GHz and $2.4 \mathrm{GHz}$ Textile Antennas for RF-Powered Body Area Networks," IEEE Access, vol. 8, pp. 133746 - 133756, 2020.

[11] C.-X. Mao, D. Vital, D. H. Werner, Y. Wu, and S. Bhardwaj, "DualPolarized Embroidered Textile Armband Antenna Array With Omnidirectional Radiation for On-/Off-Body Wearable Applications," IEEE Trans. Antennas Propag., vol. 68 no. 4, pp. 2575 - 2584, 2020.

[12] M. K. Magill, G. A. Conway, and W. G. Scanlon, "Circularly Polarized Dual-Mode Wearable Implant Repeater Antenna With Enhanced IntoBody Gain," IEEE Trans. Antennas Propag., vol. 68 no. 5, pp. 3515 3524, 2020.

[13] C. X. Mao, Y. Zhou, Y. Wu, H. Soewardiman, D. H. Werner, and J. S. Jur, "Low-Profile Strip-Loaded Textile Antenna with Enhanced Bandwidth and Isolation for Full-Duplex Wearable Applications," IEEE Trans. Antennas Propag., vol. 68 no. 9, pp. 6527 - 6537, 2020.

[14] H. Li, S. Sun, B. Wang, and F. Wu, "Design of compact single-layer textile mimo antenna for wearable applications," IEEE Transactions on Antennas and Propagation, vol. 66, no. 6, pp. 3136-3141, 2018.

[15] S. Yan, P. J. Soh, and G. A. E. Vandenbosch, "Dual-band textile mimo antenna based on substrate-integrated waveguide (siw) technology," IEEE Transactions on Antennas and Propagation, vol. 63, no. 11, pp. 4640-4647, 2015.

[16] D. Wen, Y. Hao, M. O. Munoz, H. Wang, and H. Zhou, "A compact and low-profile mimo antenna using a miniature circular high-impedance surface for wearable applications," IEEE Transactions on Antennas and Propagation, vol. 66, no. 1, pp. 96-104, 2018.

[17] A. Iqbal, A. Smida, A. J. Alazemi, M. I. Waly, N. Khaddaj Mallat, and S. Kim, "Wideband circularly polarized mimo antenna for high data wearable biotelemetric devices," IEEE Access, vol. 8, pp. 17 935-17 944, 2020.

[18] M. Wagih, G. S. Hilton, A. S. Weddell, and S. Beeby, "Dual-Band DualMode Textile Antenna/Rectenna for Simultaneous Wireless Information and Power Transfer (SWIPT)," IEEE Trans. Antennas Propag., 2021.

[19] J. Bito, R. Bahr, J. G. Hester, S. A. Nauroze, A. Georgiadis, and M. M. Tentzeris, "A Novel Solar and Electromagnetic Energy Harvesting System With a 3-D Printed Package for Energy Efficient Internet-ofThings Wireless Sensors," IEEE Trans. Microw. Theory Techn., vol. 65 no. 5, pp. $1831-1842,2017$.

[20] A. E. Abdulhadi and R. Abhari, "Multiport uhf rfid-tag antenna for enhanced energy harvesting of self-powered wireless sensors," IEEE Transactions on Industrial Informatics, vol. 12, no. 2, pp. 801-808, 2016.

[21] P. Lu, X.-S. Yang, and B.-Z. Wang, "A Two-Channel Frequency Reconfigurable Rectenna for Microwave Power Transmission and Data Communication," IEEE Trans. Antennas Propag., vol. 65 no. 12, pp. 6976 - 6985, 2017.

[22] P. Lu, C. Song, and K. M. Huang, "A Two-Port Multi-Polarization Rectenna with Orthogonal Hybrid Coupler for Simultaneous Wireless Information and Power Transfer (SWIPT)," IEEE Trans. Antennas Propag., vol. 68 no. 10, pp. 6893 - 6905, 2020.

[23] K. Dautov, M. Hashmi, G. Nauryzbayev, N. Nasimuddin, and M. A. Chaudhary, "Compact multi-frequency system design for swipt applications," International Journal of RF and Microwave Computer-Aided Engineering, vol. 31, no. 6, p. e22632, 2021.

[24] M. Wagih, G. S. Hilton, A. S. Weddell, and S. Beeby, "2.4 GHz Wearable Textile Antenna/Rectenna for Simultaneous Information and Power Transfer," in 2021 15th European Conference on Antennas and Propagation, 2021.

[25] K. Turbic, L. M. Correia, and M. Beko, "A Channel Model for Polarized Off-Body Communications With Dynamic Users," IEEE Trans. Antennas Propag., vol. 67 no. 11, pp. 7001 - 7013, 2019.

[26] M. Wagih, A. S. Weddell, and S. Beeby, "Rectennas for RF Energy Harvesting and Wireless Power Transfer: a Review of Antenna Design [Antenna Applications Corner]," IEEE Antennas Propag. Mag., vol. 62 no. 5 , pp. $95-107,2020$.

[27] K. R. Jha, Z. A. P. Jibran, C. Singh, and S. K. Sharma, "4-port mimo antenna using common radiator on a flexible substrate for sub-1ghz, sub- 6ghz 5g nr and wi-fi 6 applications," IEEE Open Journal of Antennas and Propagation, pp. 1-1, 2021

[28] C. Song, Y. Huang, P. Carter, J. Zhou, S. D. Joseph, and G. Li, "Novel Compact and Broadband Frequency-Selectable Rectennas for a Wide Input-Power and Load Impedance Range," IEEE Trans. Antennas Propag., vol. 66, 7, pp. $3306-3316,2018$.

[29] H. Sun and W. Geyi, "A New Rectenna With All-Polarization-Receiving Capability for Wireless Power Transmission," IEEE Antennas Wireless Propag. Lett., vol. 15, pp. 814 - 817, 2015.

[30] N. Ginzberg, D. Regev, G. Tsodik, S. Shilo, D. Ezri, and E. Cohen, "A full-duplex quadrature balanced rf front end with digital pre-pa selfinterference cancellation," IEEE Transactions on Microwave Theory and Techniques, vol. 67, no. 12, pp. 5257-5267, 2019.

[31] C. Song, Y. Huang, J. Zhou, P. Carter, S. Yuan, Q. Xu, and Z. Fei, "Matching Network Elimination in Broadband Rectennas for HighEfficiency Wireless Power Transfer and Energy Harvesting," IEEE Transactions on Industrial Electronics, vol. 64, 5, pp. 3950 - 3961, 2017.

[32] J. W. Massey and A. E. Yilmaz, "Austinman and austinwoman: Highfidelity, anatomical voxel models developed from the vhp color images," in 2016 38th Annual International Conference of the IEEE Engineering in Medicine and Biology Society (EMBC), 2016.

[33] H. Sun, Y. xin Guo, M. He, and Z. Zhong, "Design of a High-Efficiency 2.45-GHz Rectenna for Low-Input-Power Energy Harvesting," IEEE Antennas Wireless Propag. Lett., vol. 11, pp. 929-932, 2012.

[34] M. Wagih, A. S. Weddell, and S. Beeby, "High-Efficiency Sub-1 GHz Flexible Compact Rectenna based on Parametric Antenna-Rectifier CoDesign," in 2020 IEEE/MTT-S International Microwave Symposium (IMS), 2020.

[35] W. G. Whittow, A. Chauraya, J. C. Vardaxoglou, Y. Li, R. Torah, K. Yang, S. Beeby, and J. Tudor, "Inkjet-Printed Microstrip Patch Antennas Realized on Textile for Wearable Applications," IEEE Antennas Wireless Propag. Lett., vol. 13, pp. 71-74, 2014.

[36] N. Chahat, M. Zhadobov, S. A. Muhammad, L. L. Coq, and R. Sauleau, "60-GHz Textile Antenna Array for Body-Centric Communications," IEEE Trans. Antennas Propag., vol. 61 no. 4, pp. 1816 - 1824, 2013.

[37] M. Ur-Rehman, N. A. Malik, X. Yang, Q. H. Abbasi, Z. Zhang, and N. Zhao, "A Low Profile Antenna for Millimeter-Wave Body-Centric Applications," IEEE Trans. Antennas Propag., vol. 65 no. 12, pp. 6329 - 6337, 2017.

[38] M. Wagih, Y. Wei, A. Komolafe, R. Torah, and S. Beeby, "Reliable UHF Long-Range Textile-Integrated RFID Tag Based on a Compact Flexible Antenna Filament," Sensors, vol. 20 (12), p. 3435, 2020.

[39] L. Vallozzi, H. Rogier, and C. Hertleer, "Dual polarized textile patch antenna for integration into protective garments," IEEE Antennas and Wireless Propagation Letters, vol. 7, pp. 440-443, 2008.

[40] M. W. Abdullah, X. Fafoutis, M. Klemm, and G. S. Hilton, "Radiation pattern analysis of single and multi-antenna wearable systems," in 2016 IEEE 84th Vehicular Technology Conference (VTC-Fall), 2016, pp. 1-5.

[41] D. L. Paul, H. Giddens, M. G. Paterson, G. S. Hilton, and J. P. McGeehan, "Impact of Body and Clothing on a Wearable Textile Dual Band Antenna at Digital Television and Wireless Communications Bands," IEEE Trans. Antennas Propag., vol. 61 no. 4, pp. 2188 - 2194, 2013.

[42] K. Palmer and M. van Rooyen, "Simple broadband measurements of balanced loads using a network analyzer," IEE Transactions on Instrumentation and Measurements, vol. 55, no. 1, pp. 266 - 272, 2006.

[43] T. Q. V. Hoang, E. Séguenot, F. Ferrero, J.-L. Dubard, P. Brachat, and J.-L. Desvilles, "3D Voltage Pattern Measurement of a $2.45 \mathrm{GHz}$ Rectenna," IEEE Trans. Antennas Propag., vol. 61, 6, pp. 3354 - 3356, 2013.

[44] H. Sun and W. Geyi, "A New Rectenna With All-Polarization-Receiving Capability for Wireless Power Transmission," IEEE Antennas Wireless Propag. Lett., vol. 15, pp. 814 - 817, 2016.

[45] A. Eid, J. Hester, and M. M. Tentzeris, "A Scalable High-Gain and Large-Beamwidth mm-Wave Harvesting Approach for 5G-powered IoT," in 2019 IEEE MTT-S International Microwave Symposium (IMS), 2019.

[46] H. Sun, H. He, and J. Huang, "Polarization-Insensitive Rectenna Arrays With Different Power Combining Strategies," IEEE Antennas Wireless Propag. Lett., vol. 19, 3, pp. 492 - 496, 2020. 


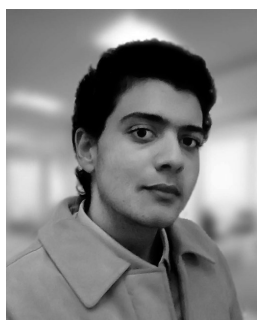

Mahmoud Wagih (GS'18, M'21) received his B.Eng. (Hons.) from the University of Southampton in September 2018, and his Ph.D. in April 2021.

In 2017 he worked as a Research Assistant at the University of Southampton. In 2018, he was a Hardware Engineering Intern at Arm, and, in 2020, a Research Intern at Arm, Cambridge, U.K. He is currently a Research Fellow at the University of Southampton, U.K., leading a Flexible Innovation Fund project on Green RF Sensing. His interests broadly cover antennas and microwave systems in energy harvesting, sensing, and wearable applications. He has 15 journal and 23 conference publications, and has delivered several invited webinars on these topics.

Dr. Wagih is a member of the Institute of Engineering and Technology (MIET). He was the recipient of the Best Undergraduate Project Prize, School Winner Doctoral Award, Best in Faculty Doctoral Research Award, and the Dean's Award, in 2018-2021, at the University of Southampton. He was selected for the IEEE International Microwave Symposium Project Connect in 2019. He received the Best Student Paper Award at the IEEE Wireless Power Transfer Conference, 2019, the Best Oral Paper at PowerMEMS, 2019, and was a Best Student Paper Finalist at IEEE WPTC, 2021. He won the MTT-S Best 3MT Presentation Prize (second place) at the IEEE Microwave Week, 2020, and was a session co-chair at the European Conference on Antennas and Propagation, 2021. He acts as a reviewer for 9 TRANSACTIONS and journals.

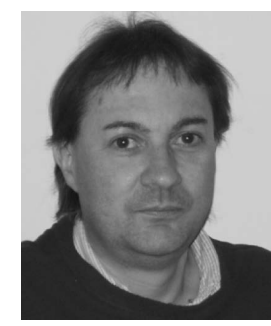

Geoffrey S. Hilton received the B.Sc. degree from the University of Leeds, Leeds, U.K., in 1984, and the Ph.D. degree from the University of Bristol, Bristol, U.K., in 1993, for research on the design and finite-difference time-domain modeling of printed antenna elements.

$\mathrm{He}$ is a Senior Lecturer with the University of Bristol. His current research interests include practical antenna and system design for a variety of communications and radar applications such as ground penetrating radar, performance evaluation of antennas in mobile radio, electrically small elements, active/tuneable elements, and vehicle-mounted conformal antennas.

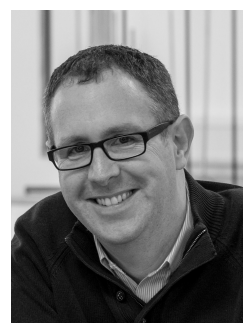

Alex S. Weddell (GS'06-M'10) received the M.Eng. degree (1st class honors) and Ph.D. in electronic engineering from the University of Southampton, U.K., in 2005 and 2010.

His main research focus is in the areas of energy harvesting and energy management for future Internet of Things devices. He has over 14 years' experience in design and deployment of energy harvesting systems, and has published around 55 peer-reviewed papers in the area. He is currently a Lecturer in the Center for Internet of Things and Pervasive Systems at the University of Southampton, and is involved with three projects funded by EPSRC, EU Horizon 2020 and Clean Sky 2.

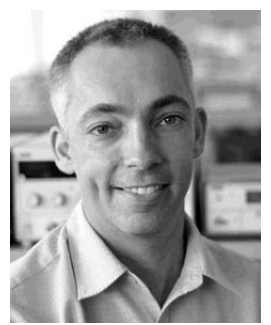

Steve Beeby received the B.Eng. (Hons.) degree in mechanical engineering from the University of Portsmouth, Portsmouth, U.K., in 1992, and the $\mathrm{Ph} . \mathrm{D}$. degree in MEMS resonant sensors from the University of Southampton, Southampton, U.K., in 1998.

$\mathrm{He}$ is currently the Head of the Smart Electronic Materials and Systems Research Group and leads the U.K.'s E-Textiles Network. He is currently leading three U.K. funded research projects and has received over $£ 16$ million research funding. $\mathrm{He}$ is a $\mathrm{Co}-$ Founder of Perpetuum Ltd., a University spin-out based upon vibration energy harvesting formed in 2004, Smart Fabric Inks Ltd., and D4 Technology Ltd. He has co-authored/edited four books including Energy Harvesting for Autonomous Systems (Artech House, 2010). He has given 25 invited talks and has over 300 publications and 9 patents. He has an h-Index of 53. His current research interests focus on energy harvesting, e-textiles and the use of energy harvesting in wearable applications.

Prof. Beeby is the recipient of a prestigious RAEng Chair in Emerging Technologies and has previously been the recipient of two prestigious EPSRC Research Fellowships to investigate the combination of screen-printed active materials with micromachined structures and textiles for energy harvesting and was also awarded a Personal Chair in 2011. He is currently the Chair of the International Steering Committee for the PowerMEMS Conference series. 